\title{
ANALYSIS OF DUAL-ENERGY X-RAY ABSORPTIOMETRY IMAGES USING COMPUTER VISION METHODS
}

\author{
N. Kirilov* \\ Faculty of Mathematics and Informatics, Sofia University, Sofia, Bulgaria
}

\begin{abstract}
PURPOSE: Dual-energy x-ray absorptiometry (DXA) is the "golden standard" for diagnosing osteoporosis. Its analyzing algorithm (software) makes it possible to distinguish the bone from the soft tissue. Until now there are only attempts to process and acquire images using automatic segmentation with convolutional neural networks $(\mathrm{CNN})$. Machine reconstruction and precise specific models of anatomic structures from medical images could be accomplished using computer vision. The objective of the current work is to introduce the potential of the two computer methods and their application in the diagnostic DXA analysis.

METHODS: DXA generates a report in the DICOM format which includes patient data (age, gender, height, weight, bone mineral density, $\mathrm{T}$-score and Z-score) and an image of the scanned spine as well as the region of interest (ROI). The CNN methods are based mainly on intermediate analysis. The learning of the segmentation of CNN by generating segmentation labels using simple heuristic is done using computer vision. The functions of the loss and the architecture of the CNN is then determined. In that manner the right analysis of the existing medical image is made possible.

RESULTS: The computer library OpenCV is the way to realize a model for the assessment of a DXA analysis. The library is available for Python programming language. The library has functions for the extraction of colour objects, image smoothing, Canny's edge detector, Hough transform and methods for work with contours.

CONCLUSIONS: The detection and extraction of images is fundamental for the analysis of DXA which is a step forward in the precision of the in-vivo diagnostic of the bone.
\end{abstract}

Key words: Dual-energy x-ray absorptiometry (DXA), convolutional neuronal networks, computer vision, diagnostic analysis

\section{PURPOSE}

The analysis of medical images requires a combination of interdisciplinary fields, including electrical, computer and biomedical engineering, as well as other fields (1). Despite the independent development of medical imaging and computer vision, the merging of these two sciences holds promising results. Scientific papers focused on the analysis of medical images with computer vision were presented at the European Conference on Computer Vision (ECCV). In 2006, a seminar on "Approaches of computer vision to the analysis of medical images" was held in Graz, Austria (2). The application of computer vision

\footnotetext{
*Correspondence to: Nikola Kirilov, Sofia University, Faculty of Mathematics and Informatics, E-mail address: kirilov_9@abv.bg, Telephone number: 0882107052
}

techniques for the analysis of medical images is used for both clinical evaluation and operations. Automated reconstruction of precise specific models of anatomical structures from medical images is becoming essential for surgical procedures, disease research, and for clinical evaluation of the effectiveness of therapy $(3,4)$. For example, in minimally invasive procedures, neurosurgeons often use image-related navigation aids to locate the field of operation (5). Therefore, the extraction of anatomical models from images by computer vision is a key element of emerging surgical techniques (6).

The only DXA image analysis has been done with a Convolutional Neural Network (CNN) (7). This methodology is based on intermediate analyzes. They can improve classification efficiency in the context of specific medical 
applications, as they are trained by databases that are usually available in the medical image. Interim analyzes incorporate prior knowledge of how pathology is imaged on DXA and provides important guidance for the network. The goal is to automatically segment the marked body parts of the DXA image with a Convolutional Neural Network (CNN). The segmenting CNN receives the DXA image at the input and produces six different channels with the same dimension as the input, where each channel corresponds to the six marked parts. Because DXA scanning of the whole body is homogeneous, segmentation labels for some parts of the body can be produced with a series of simple heuristics. These labels, while not perfect, are good enough to train the segmenting $\mathrm{CNN}$, and in many cases, the trained CNN produces visually better segmentation. To date, no specific analyzer of DXA test results by computer vision has been developed.

Dual-energy X-ray absorptiometry (DXA) or double energy X-ray absorptiometry is an Xray method for diagnosis of osteoporosis accepted and established worldwide as the "gold standard". Different risk assessment tools could be used to decide if the patients should undergo DXA scans (8-13). Bone mineral density is measured in $\mathrm{g} / \mathrm{cm} 2$ and the $\mathrm{T}$-score and Z-score for this value are calculated. To obtain a qualitative and accurate DXA assessment, a number of conditions must be met. One of them and most importantly, is the correct positioning of the patient under the $\mathrm{X}$-ray and the correct setting of the field of interest (ROI - a region of interest) for calculating bone density. The first 4 lumbar vertebrae (L1-L4) are of interest for the study of bone density by the DXA method. They must be positioned in this field under strictly defined rules in order to obtain research with maximum quality and value. Bone mineral density is determined for each vertebra individually and for all as a final result. If some of the conditions for qualitative research are not met, the researcher may receive compromised results. An example of such a case is when the spine has a large curvature, it cannot be placed correctly in the region of interest and the results of the bone mineral density are false, so making a correct diagnosis is difficult. The correct diagnosis is important to make a decision about the treatment (14, $15)$.
The DXA study generates a report, which is in DICOM format and includes patient data (age, sex, height, weight, bone mineral density, Tscore and Z-score) and an image of the scanned spine, as well as location of the field of interest.

The objective of the current work is to introduce the potential of the two computer methods and their application in the diagnostic DXA analysis.

\section{METHODS}

The following methods of computer vision were used to perform the set tasks: extraction of colored objects from an image, Gaussian blurring, detection of boundaries in an image (Canny's algorithm), Hough transformation and contour detection. The file format of the examined data was the DICOM standard.

Each DICOM report from DXA scan includes:

- patient data (age, gender, height, weight, bone mineral density (BMD), T-score and Z-score)

- image of the scanned spine as well as the region of interest (ROI).

The program implementation of the methods was performed in Python programming language through the libraries PyDICOM, OpenCV, NumPy and PIL. The Tkinter module was used to create the graphical interface of the application.

Once detected, each of the features can be extracted from the DXA scan image. This extraction often requires the use of a large amoun of resources and the result is known as a descriptor or attribute vector.

Feature detection involves methods for calculating image information and making decisions at each point in the image for the presence of a feature of a particular type. Feature extraction is a dimensional reduction process that starts with the original set of output variables, from which less is constructed for accessible processing and sufficiency to accurately and completely describe the original data set. Sign extraction is used in machine learning, image recognition and image processing. It starts with the initial set of data from which we obtain secondary derivative values (signs), which are considered to be informative and unambiguous. This achieves a consistent process of machine learning and generalization of the steps and 
leads to a better human interpretation of the data. One of the most important areas of application is image processing, in which algorithms are used to detect and isolate a variety of desired areas or shapes (characteristics) of a digital image. It is widely used in Optical Character Recognition (OCR) (15).

\section{RESULTS}

The Open CV computer library is used for the software implementation of the DXA analysis. It is available in Python under the name "cv2" and is included in the program with import cv2. The library uses functions for program implementation of the method for extracting colored objects, the method for image blurring, the Canny algorithm, the Hough transformation and the methods for working with contours. The extraction of colored objects is required in the evaluation for the correct location of the region of interest. Initially, the color space of the image is changed with cvtColor (input_image, flag). Then, by setting the color boundary values of the field of interest, the inRange (input_image (HSV), lower_bound, upper_bound) function is used by OpenCV, extracting only its pixels. The bitwise_and function (input_image, output_image, mask = mask) masks the field of interest from the original image and extracts it. The program implementation of the process is part of the program method of the analyze_roi () model.

Gaussian blur is applied in the computer model to evaluate DXA analysis in order to preprocess the image and to reduce noise before applying other methods. It was used before Canny's algorithm and Hough's transformation. In Python, Gaussian blur is implemented with the function from the OpenCV GaussianBlur library (input_image, (matrix size)) and is performed in the program method of the model analyze_angle () and analyze_roi (). The result is an image with a lower noise level and clearer borders. This improves the performance of Canny's algorithm, which detects boundaries more efficiently, which in turn is needed for better Hough transformation results. The Canny algorithm is the main method for detecting boundaries in the image used in the DXA analysis evaluation model. The application of the algorithm is performed in the analysis to calculate the distortion angle before the Hough transformation and before the detection of the contours of the field of interest in the assessment of the correct location of the field of interest.

Hough transformation, as one of the most effective methods for detecting lines in an image, is used in the DXA analysis evaluation model. The detection of straight lines is performed when calculating the angle of distortion. The aim is to find the lines corresponding to the vertebrae and to calculate the angle of curvature. The program implementation of the method in Python is performed through the function from the OpenCV HoughLines library (input_image, $\rho$, $\theta$, threshold) and is part of the program method of the analyze_angle () model.

\section{DISCUSSION}

Using the methods of computer vision algorithm was developed to analyze DXA scan images. Based on the developed application for analysis of DXA images, important information can be derived about the angle of curvature in the lumbar region in people who have performed a DXA scan. The application may be useful for conducting large-scale studies to determine the incidence of spinal curvature from DXA images, as this type of study involves less radiation than a standard Xray. Additionally, the application helps to correctly interpret the DXA scans, as it provides information about the angle of lumbar curvature. To date, no such DXA image analyser based on computer vision has been developed, and this thesis lays the foundations for a significant discovery. This application can be used in the future in medical practices in order to improve the diagnosis of osteoporosis.

\section{CONCLUSIONS}

The detection and extraction of images is fundamental for the analysis of DXA which is a step forward in the precision of the in-vivo diagnostic of the bone.

\section{REFERENCES}

1. Sun Z, Ng KH, Ramli N. Biomedical imaging research: a fast-emerging area for interdisciplinary collaboration. Biomed Imaging Interv J, 7(3): 21. doi:10.2349/biij.7.3.e2., 2011

2. Beichel, Reinhard R., Sonka, Milan. Computer Vision Approaches to Medical Image Analysis. Second International ECCV Workshop, , Graz, Austria, May 12, Revised Papers,CVAMIA, 2006 
3. Lundervold AS, Lundervold A. An overview of deep learning in medical imaging focusing on MRI. Z Med Phys, 29(2):102-127, 2019

4. European Society of Radiology (ESR). Medical imaging in personalised medicine: a white paper of the research committee of the European Society of Radiology (ESR). Insights Imaging.6(2):141-155, 2015

5. Mezger U, Jendrewski C, Bartels $M$. Navigation in surgery. Langenbecks Arch Surg, 398(4):501-514, 2013

6. Grimson E., Golland P. Analyzing Anatomical Structures: Leveraging Multiple Sources of Knowledge. In: Liu Y., Jiang T., Zhang C. (eds) Computer Vision for Biomedical Image Applications. Lecture Notes in Computer Science, Springer, Berlin, Heidelberg. vol 3765, CVBIA, 2005

7. Amir Jamaludin, Timor Kadir. Predicting Scoliosis in DXA Scans Using Intermediate Representations5th International Workshop and Challenge, CSI 2018 Held in Conjunction with MICCAI Granada, Spain, September 16, Revised Selected Papers: 15-28, 2018

8. Kirilova E, D Tcherkezov, V Gonchev, Z Zheleva OSIRIS index for assessment of the osteoporosis risk in postmenopausal women. National conference Science and Society, Kardjali, RKR print OOD ISSN 1314-3425:95-99, 2019

\section{KIRILOVN.}

9. Madjarova R., E.Kirilova, T.Petranova, M.Nikolova Evaluation of Indipendent care activities in adult patient with osteoporosis, Science and Technologie,: Medical biology studies,clinical studies, social medicine and health care, 1, Vol VIII:131-136, 2018

10.Zheleva Z, Kirilova E, Popov I. Selfassessment tool for the risk of Osteoporosis in postmenopausal women. Science \& Technologies: Medical biology studies, clinical studies, social medicine and health care, Vol IX: 111-115, 2019

11.Kirilova E, D Tcherkezov, V Gonchev, Z Zheleva. Study of the ORAI (osteoporosis risk assesment) index for assessing the risk of osteoporosis in menopause women National conference Science and Society, Kardjali, RKR print OOD ISSN 1314-3425: 91-94, 2019

12.Kirilova E., I.Popov. Osteoporosis prevalence and prevention ISBN: 978-9549374-14-8, 2019

13.Kirilova E. Radiofrequency echographic multi spectrometry(REMS) for axial skeleton Trakij OOD, ISBN: 978-9549374-15-5, 2019

14. Vladeva S, Kirilova E. Bisphosphonate therapy of patient with multifocal reflex sympathetic dystrophy and general osteoporosis. Bone ,48( 2): P S231, 2011

15.Alpaydin E. Introduction to Machine Learning. London: The MIT Press. p. 110. 2010, ISBN 978-0-262-01243-0, Retrieved 4 February 2017. 